\title{
1,2,4-三唑-3-硫醚衍生物的合成、晶体结构与神经氨酸酶抑制活性
}

\author{
何 梅 ${ }^{a}$ 贺超凡 ${ }^{a}$ 刘 玲 ${ }^{a}$ 叶 姣*,a 胡艾希*,a \\ 陈云 ${ }^{a}$ 许律捷 ${ }^{b}$ 刘艾林 ${ }^{b}$ \\ ( ${ }^{a}$ 湖南大学化学化工学院 长沙 410082$)$ \\ $\left({ }^{b}\right.$ 中国医学科学院北京协和医学院药物研究所 北京 100050)
}

\begin{abstract}
摘要 设计并合成了一系列 1,2,4-三唑-3-硫梄衍生物, 目标化合物的化学结构经 ${ }^{1} \mathrm{H} N \mathrm{NM} 、{ }^{13} \mathrm{C} N \mathrm{NM}$ 、质谱和元素分析 确证; 采用单晶 $\mathrm{X}$ 射线衍射法测定了(E)-4-(4-差基-3-甲氧基苯基亚甲氨基)-5-乙基-4H-1,2,4-三坐-3-丙硫醚(1c)的晶体 结构. 目标化合物体外神经氨酸酶(Neuraminidase, NA, H1N1)抑制活性测试结果表明, 大部分化合物 1 具有较好的 NA 抑制活性，其中 ( $E$ )-4-(4-羟基-3-甲氧基苯基亚甲氨基)-5-乙基-4H-1,2,4-三唑-3-乙硫醚(1b)和 $\mathbf{1}$ 的 NA 抑制活性最佳，其 $\mathrm{IC}_{50}$ 值分别为(6.86 2.08$)$ 和 $(9.1 \pm 1.56) \mu \mathrm{g} / \mathrm{mL}$.
\end{abstract}

关键词 1,2,4-三唑-3-硫醚衍生物; 合成; 晶体结构; 神经氨酸酶抑制活性

\section{Synthesis, Crystal Structure and Neuraminidase Inhibitory Activity of 1,2,4-Triazole-3-sulfide Derivatives}

\author{
$\mathrm{He}, \mathrm{Mei}^{a}$ \\ He, Chaofan ${ }^{a}$ \\ Liu, Ling ${ }^{a}$ \\ Ye, Jiao*,a \\ $\mathrm{Hu}, \mathrm{Aixi}^{*, a}$ \\ Chen, Yun $^{a}$ \\ $\mathrm{Xu}$, Lujie $^{b}$ \\ Liu, Ailin ${ }^{b}$ \\ ( ${ }^{a}$ College of Chemistry and Chemical Engineering, Hunan University, Changsha 410082) \\ ( ${ }^{b}$ Institute of Materia Medica, Chinese Academy of Medical Sciences and Peking Union Medical College, Beijing 100050)
}

\begin{abstract}
A series of 1,2,4-triazole-3-sulfide derivatives were designed and synthesized. Their chemical structures were confirmed by ${ }^{1} \mathrm{H}$ NMR, ${ }^{13} \mathrm{C}$ NMR, MS and elemental analysis. The crystal structure of $(E)$-4-(4-hydroxy-3-methoxyphenylmethyleneamino)-5-ethyl-4H-1,2,4-triazole-3-propylsulfide (1c) was determined by X-ray diffraction analysis. The preliminary assay of neuraminidase (NA, H1N1) inhibitory activity in vitro showed that most of compound $\mathbf{1}$ has more potent NA inhibitory activity. Among them, compounds (E)-4-(4-hydroxy-3-methoxyphenyl-methyleneamino)-5-ethyl-4H-1,2,4-triazole3-ethylsulfide (1b) and $\mathbf{1 c}$ showed the best inhibitory activity with $\mathrm{IC}_{50}$ values of $(6.86 \pm 2.08)$ and $(9.1 \pm 1.56) \mu \mathrm{g} / \mathrm{mL}$, respectively.

Keywords 1,2,4-triazole-3-sulfide derivative; synthesis; crystal structure; neuraminidase inhibitory activity
\end{abstract}

流感是一种爆发频繁、致死率高、严重威胁人类健 康的疾病 ${ }^{[1-2]}$. 目前抗流感药物根据其作用机理的不同 主要分为 M2 离子通道抑制剂(金刚烷胺和金刚乙胺)、 神经氨酸酶(Neuraminidase, NA)抑制剂(扎那米韦、奥司 米韦、帕拉米韦、拉尼米韦)、核糖核酸(RNA)聚合酶抑 制剂(法匹拉韦)、血凝素(HA)抑制剂(阿比多尔)和帽状 结构(Cap)依赖型核酸内切酶抑制剂(巴洛沙韦 $)^{[3-6]}$. 其 中, 金刚烷胺和金刚乙胺是最早研制成功的抗流感药 物, 只能抑制甲型流感病毒的 M2 离子通道, 临床使用
已有四十余年, 由于其严重的抗药性已不再推荐用于治 疗流感 ${ }^{[7]}$. 神经氨酸酶抑制剂(NAIs) 是第二代主流的抗 流感药物, 主要是通过抑制呼吸道病毒表面神经氨酸酶 的活性，从而阻断病毒的复制和释放，达到抗流感病毒 的作用, 是目前使用最为广泛的治疗和预防流感的药 物 ${ }^{[8]}$. 虽然流感病毒抗原极易变异, 但所有 $\mathrm{A}$ 型和 $\mathrm{B}$ 型 流感病毒的 NA 活性位点具有高度保守性 ${ }^{[9-10]}$, 所以 NA 一直是研发抗流感药物的理想靶标. 目前已上市的 4 个 NAIs，扎那米韦(Zanamivir)、奥司他韦(Oseltamivir)、帕

* Corresponding authors. E-mail: yejiao@hnu.edu.cn; axhu@hnu.edu.cn.

Received March 5, 2020; revised May 21, 2020; published online May 27, 2020.

Project supported by the Natural Science Foundation of Hunan Province (No. 2019JJ40030).

湖南省自然科学基金(No. 2019JJ40030)资助项目. 
拉米韦(Peramivir)和拉尼米韦(Laninamivir), 均具有优 异的 NA 抑制活性和整体抗病毒活性, 但该类药物的结 构中含有多个手性中心, 结构复杂, 合成困难, 所需原 料昂贵, 生产成本高; 同时随着 NAIs 药物的广泛使用, 耐药病毒迅速出现 ${ }^{[11-12]}$, 因此, 急需研究开发结构新颖 的新型抗流感药物, 以应对未来的流感大爆发.

近年来, 随着大量具有低毒性和较高生物活性的含 氮杂环化合物被报道, 含氮杂环已成为新药创制研究的 热点领域之一 ${ }^{[13-20]}$. 其中 $1,2,4$-三唑硫酮(硫醇或硫醚) 衍生物是一类具有广泛生物活性的含氮杂环化合物, 如

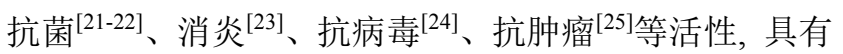
开发新药和新的药理活性的价值. 例如: Sharma 等 ${ }^{[26]}$ 合 成的 1,2,4-三唑硫醇亚胺类衍生物具有抑菌作用, 其中 化合物 $\mathbf{A}$ (图 1)对大肠杆菌(Escherichia coli)抑菌值为 (10.2 \pm 0.24$) \mathrm{mm}$, 对革兰氏阳性菌(Streptococcus pneumoniae)和真菌(Aspergillus niger)抑菌值分别为(11.28土 $0.25)$ 和(17.24 \pm 0.21$) \mathrm{mm}$ [阳性对照药环丙沙星对大肠 杆菌抑菌值为 $(19.67 \pm 0.58) \mathrm{mm}$, 对革兰氏阳性菌抑菌 值为 $(20.18 \pm 0.16) \mathrm{mm}$, 阳性对照药氟康唑对真菌抑菌 值为 $(20.31 \pm 0.13) \mathrm{mm}$. Liu 等 ${ }^{[27]}$ 设计并合成的含有 1,2,4-三唑硫醚亚胺的化合物 $\mathbf{B}$ (图 1) 具有抗 HIV-1 III $_{\mathrm{B}}$ 活 性, 其 $\mathrm{EC}_{50}$ 值为 $17.4 \mu \mathrm{mol} / \mathrm{L}$. Kamel 等 ${ }^{[28]}$ 研究了 1,2,4三唑硫醇类衍生物的抗癌活性, 其中化合物 $\mathbf{C}$ (图 1)对 $\mathrm{HEPG} 2$ 癌细胞表现出了 $\mathrm{nmol} / \mathrm{L}$ 级的抑制活性, 其 $\mathrm{IC}_{50}$ 值为 $53 \mathrm{nmol} / \mathrm{L}$. El-Husseiny 等 ${ }^{[29]}$ 以菜普生为骨架, 设计 合成的 1,2,4-三唑硫酮亚胺类衍生物具有抗炎作用, 其<smiles>O=C(O)c1ccc(-c2nnc(S)n2/C=C/C(=O)c2ccccc2O)cc1</smiles>

A<smiles>CC(=O)Nn1c(S)nnc1-c1ccncc1</smiles>

C<smiles>CCc1n[nH]c(=S)n1/N=C/c1ccc(O)c(OC)c1</smiles>

E<smiles>[B]Oc1ccc(/C=N/n2c(SCc3cccc(C#N)c3)nnc2-c2ccco2)cc1OC</smiles>

B<smiles>COc1ccc2cc(C(C)c3n[nH]c(=S)n3/N=C/c3ccc(O)cc3)ccc2c1</smiles><smiles>COc1cc(/C=N/n2c(C(C)O)n[nH]c2=S)ccc1O</smiles>

图 1 1,2,4-三唑硫酮(硫醇或硫醚)衍生物

Figure 1 1,2,4-Triazolethione (thiol or thioether) derivatives
中化合物 $\mathbf{D}$ (图 1)对 COX-2 酶有优良的抑制活性，其 $\mathrm{IC}_{50}$ 为 $0.40 \mu \mathrm{mol} / \mathrm{L}$.

1,2,4-三唑硫酮(硫醇或硫醚)衍生物除具有以上生 物活性外，还具有良好的 NA 抑制活性，本课题组 ${ }^{\left[{ }^{[0]}\right.}$ 在 前期研究中, 通过骨架跃迁方法设计并合成了一系列 1,2,4-三唑-3-硫酮衍生物, 大部分具有良好的神经氨酸 酶(H1N1)抑制活性, 其中活性最好的化合物 $\mathbf{E}$ 和 $\mathbf{F}$ (图 $1)$ 的 $\mathrm{IC}_{50}$ 值分别为 $(14.97 \pm 0.70)$ 和(14.68 \pm 0.49$) \mu \mathrm{g} / \mathrm{mL}$. 通过分子对接分析发现，化合物 $\mathbf{E}$ 与 NA (PDA entry: 3TI6)的活性空腔有两个作用位点,一个是苯环上的 4-OH-3- $-\mathrm{OCH}_{3}$ 类似于奥司他韦羧酸的羧基, 能与 $\operatorname{Arg} 118, \operatorname{Arg} 292$ 和 $\operatorname{Arg} 371$ 形成多个氢键作用, 另一个是 $1,2,4-$ 三唑环上的两个氮原子与 Glu119 具有氢键作用 (图 2).
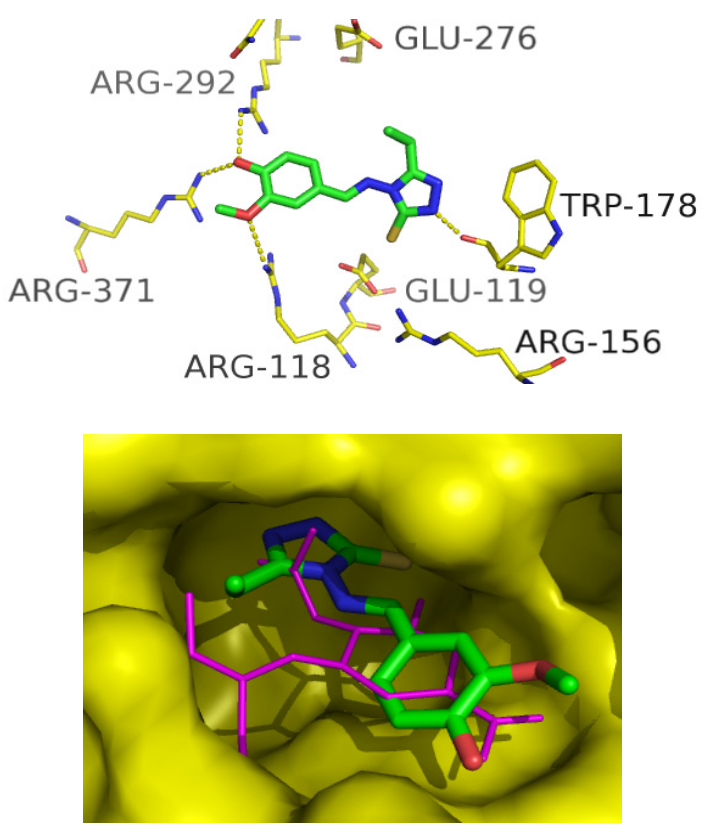

图 2 化合物 $\mathbf{E}$ 与 $\mathrm{A} / \mathrm{H} 1 \mathrm{~N} 1-\mathrm{NA}$ (3TI6)的分子对接模型

Figure 2 Interaction models of $\mathbf{E}$ with A/H1N1-NA (3TI6) The top picture shows binding models of $\mathbf{E}$ with A/H1N1-NA. The $\mathrm{H}$-bond interactions are depicted as yellow dashed lines. The bottom picture shows comparison of the interactions of $\mathbf{E}$ and Oseltamivir acid with A/H1N1-NA by structural superposition

为进一步提高该类化合物与 NA 活性位点的结合能 力, 本工作在化合物 $\mathbf{E}$ 的基础上, 将硫酮转变为硫醚, 通过引入疏水性基团增加分子与靶点的疏水作用. 因此 在目标分子的 $\mathrm{R}$ 位置引入不同的烷基和芳基，探究取代 基的大小、位阻和极性对 NA 抑制活性的影响，设计并 合成了 11 个新型 $1,2,4$-三唑硫醚亚胺衍生物; 然后从中 篮选出活性较佳的 2 个化合物, 将其 $\mathrm{N}=\mathrm{C}$ 还原成 $\mathrm{NH}-\mathrm{CH}$ ，增大分子的柔性，设计合成了 2 个新型 1,2,4三唑硫醚胺类衍生物(Scheme 1), 考察化合物刚性与柔 性对活性的影响, 并对所有目标化合物体外 NA 抑制活 
性进行评估. 目标化合物的合成路线见 Scheme 2.<smiles>[R]CSc1nnc(CC)n1/N=C\c1ccc(O)c(OC)c1</smiles>

Previous work<smiles>[R]Sc1nnc(CC)n1NC(=O)c1ccc(O)c(OC)c1</smiles>

图式 1 目标化合物 $\mathbf{1}$ 和 $\mathbf{2}$ 的设计

Scheme 1 Design strategy of the target compounds $\mathbf{1}$ and $\mathbf{2}$

\section{1 结果与讨论}

\section{1 目标化合物 $1 \mathrm{a} \sim 1 \mathrm{k}$ 和 $2 \mathrm{~b} \sim 2 \mathrm{c}$ 的合成}

化合物 $\mathbf{E}$ (按文献 ${ }^{[30]}$ 合成)在碱作用下，经硫烷基化 反应得目标化合物 $\mathbf{1 a} \sim 1 \mathbf{k}$, 收率为 $39.7 \% \sim 78.7 \%$. 用 嗍氢化钠将化合物 $1 \mathrm{~b}$ 和 $1 \mathrm{c}$ 的 $\mathrm{N}=\mathrm{C}$ 还原成 $\mathrm{N}-\mathrm{C}$ 得目 标化合物 $\mathbf{2 b}$ 和 $\mathbf{2 c}$ ，收率为 $81.0 \% \sim 82.0 \%$. 所合成的目

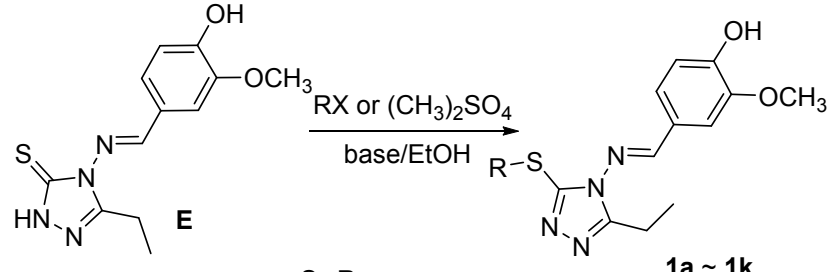<smiles>[R]Sc1nnc(CC)n1NCc1ccc(O)c(OC)c1</smiles>

1a: $\mathrm{R}=\mathrm{CH}_{3} ; \mathbf{1 b}, \mathbf{2 b}: \mathrm{R}=\mathrm{C}_{2} \mathrm{H}_{5} ; \mathbf{1 c}, \mathbf{2 c}: \mathrm{R}=n-\mathrm{Pr} ; \mathbf{1 d}: \mathrm{R}=i-\mathrm{Pr} ; \mathbf{1 e}: \mathrm{R}=n$ $\mathrm{C}_{4} \mathrm{H}_{9} ; 1$ ff: $\mathrm{R}=$ sec $-\mathrm{C}_{4} \mathrm{H}_{9} ; 1$ g: $\mathrm{R}=n-\mathrm{C}_{5} \mathrm{H}_{11} ; 1$ h: $\mathrm{R}=\mathrm{CH}\left(\mathrm{CH}_{2} \mathrm{CH}_{3}\right)_{2} ; 1 \mathrm{i}: \mathrm{R}=$ $\mathrm{CH}_{2} \mathrm{COOH} ; \mathbf{1 j :} \mathrm{R}=4-\mathrm{O}_{2} \mathrm{NC}_{6} \mathrm{H}_{4} \mathrm{CH}_{2} ; \mathbf{1 k}: \mathrm{R}=4-\mathrm{NCC}_{6} \mathrm{H}_{4} \mathrm{CH}_{2}$

图式 2 目标化合物 $\mathbf{1}$ 和 2 的合成路线

Scheme 2 Synthetic routes of target compounds 1 and 2 标化合物均通过 ${ }^{1} \mathrm{H} N M R 、{ }^{13} \mathrm{C} N M R 、 M S$ 和元素分析 确证结构.

\section{2 化合物 $1 \mathrm{c}$ 晶体结构解析}

(E)-4-(4-羟基-3-甲氧基苯基亚甲氨基)-5-乙基-4H1,2,4-三唑-3-丙硫醚(1c)单晶体结构如图 3 所示, 氢键如 图 4 所示.

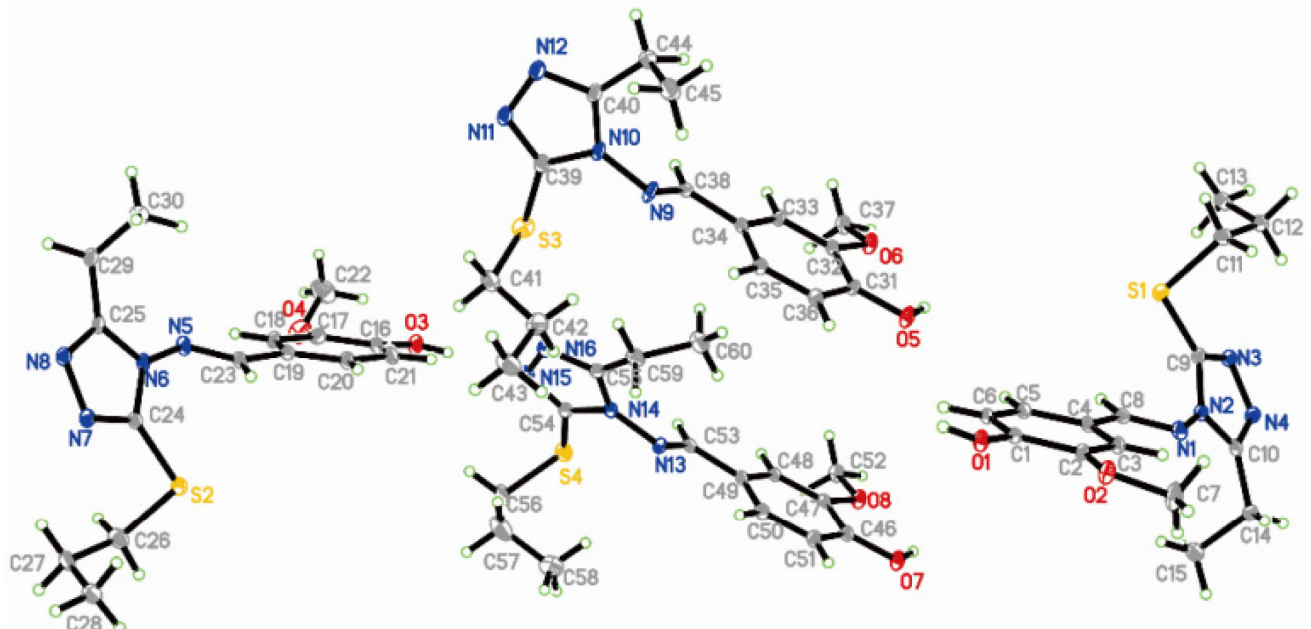

图 3 化合物 $1 \mathrm{c}$ 的单晶结构图

Figure 3 Single crystal structure of compound 1c

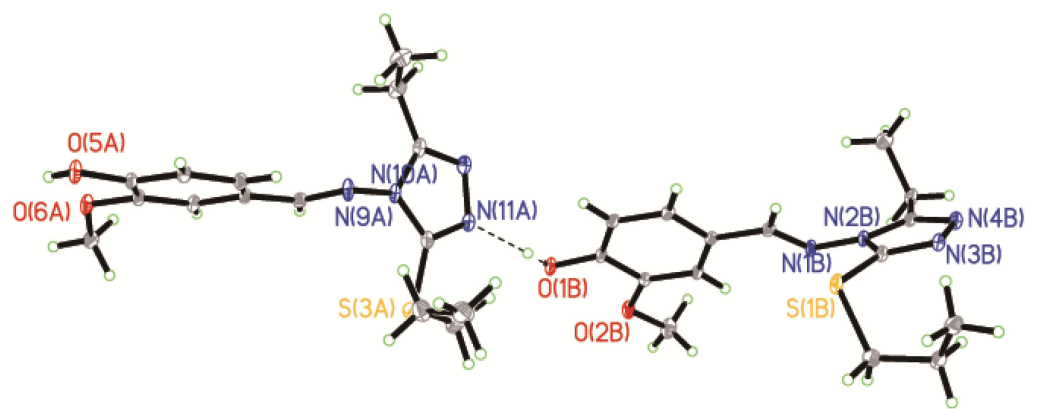

图 4 化合物 $1 \mathrm{c}$ 的晶体中的分子间氢键作用图

Figure 4 Intermolecular hydrogen bonding in the crystal of compound 1c 
化合物 1c 晶体数据存于英国剑桥数据中心, $\mathrm{CCDC}$ 号为 1923378 , 分子式为 $\mathrm{C}_{15} \mathrm{H}_{20} \mathrm{~N}_{4} \mathrm{O}_{2} \mathrm{~S}, M_{\mathrm{r}}=320.41$, m.p. $119 \sim 121{ }^{\circ} \mathrm{C}$. 属于三斜晶系, 空间群为 $P-1$, 每个单元 含有 2 个分子, 晶胞参数为: $a=1.0601(8) \mathrm{nm}, b=$ $1.5344(11) \mathrm{nm}, c=2.0476(9) \mathrm{nm} ; Z=2, V=3.129(3) \mathrm{nm}^{3}$, $D_{\mathrm{c}}=1.360 \mathrm{Mg} / \mathrm{m}^{3}, S=1.042, F(000)=1360, \mu=0.220$ $\mathrm{mm}^{-1}$.

化合物 1c 只有一种硫醚式结构, 说明化合物 $\mathbf{E}$ 与 烷基化试剂在碱性条件下的反应是以硫烷基化反应为 主; 另外, 由于丙基柔性较强, 易发生扭转, 导致化合 物 1c 具有四种构象, 且每种构象分子的苯环与 1,2,4-三 唑环的二面角大小有所差别, 第一种构象至第四种构象 的二面角分别为 $56.62^{\circ}, 64.02^{\circ}, 86.98^{\circ}$ 和 $53.95^{\circ}$; 化合物 $1 \mathrm{c}$ 有两个碳硫键, $\mathrm{S}(1)-\mathrm{C}(9)$ 和 $\mathrm{S}(1)-\mathrm{C}(11)$ 的键长分别 0.174 和 $0.183 \mathrm{~nm}$, 相比于 $\mathrm{C}=\mathrm{S}(0.164 \mathrm{~nm})$ 的标准键长 增大, 且与 $\mathrm{C}-\mathrm{S}(0.182 \mathrm{~nm})^{[31]}$ 的标准键长更接近, 说明 $\mathrm{C}$ 与 $\mathrm{S}$ 之间为单键, 也进一步证明了化合物 $1 \mathrm{c}$ 为硫醚式 结构(图 3); 化合物 $\mathbf{1 c}$ 的晶体能通过苯环上的 $\mathrm{OH}$ 与相 邻分子的 1,2,4-三唑环上 1 位的 $\mathrm{N}$ 之间形成分子间氢键 而稳定(图 4), 但是由于化合物 $1 \mathrm{c}$ 有四种构象, 各类分 子间所形成的氢键有差别, 其氢键距离及角度数据见表 1. 另外, 分子结构中 $\mathrm{N}=\mathrm{C}$ 为 $E$ 式构型.

表 1 化合物 $1 \mathrm{c}$ 的氢键 $\left[\mathrm{nm}\right.$ 和 $\left.\left(^{\circ}\right)\right]$

Table 1 Hydrogen bonds [nm and $\left(^{\circ}\right)$ ] of compound $\mathbf{1 c}$

\begin{tabular}{ccccc}
\hline $\mathrm{D}-\mathrm{H} \cdots \mathrm{A}$ & $d(\mathrm{D}-\mathrm{H})$ & $d(\mathrm{H} \cdots \mathrm{A})$ & $d(\mathrm{D} \cdots \mathrm{A})$ & $\angle(\mathrm{DHA})$ \\
\hline $\mathrm{O}(1)-\mathrm{H}(1) \cdots \mathrm{N}(11)$ & 0.0820 & 0.1870 & 0.2687 & 173.28 \\
$\mathrm{O}(3)-\mathrm{H}(3) \cdots \mathrm{N}(15)$ & 0.0820 & 0.1936 & 0.2750 & 171.45 \\
$\mathrm{O}(5)-\mathrm{H}(5 \mathrm{~A}) \cdots \mathrm{N}(8)$ & 0.0820 & 0.1972 & 0.2721 & 151.48 \\
$\mathrm{O}(7)-\mathrm{H}(7) \cdots \mathrm{N}(4)$ & 0.0820 & 0.1954 & 0.2699 & 150.71 \\
\hline
\end{tabular}

\section{3 生物活性}

以扎那米韦(zanamivir)为阳性对照药, 评估了所有 目标化合物的体外 NA(H1N1)抑制活性, 并与化合物 $\mathbf{E}$ 和 $\mathbf{F}$ 进行了对比, 如表 2 所示. 从 $\mathrm{IC}_{50}$ 值看在化合物 $\mathbf{E}$ 基础上进一步改造的化合物 $\mathbf{1}$ 大部分表现出更优的 NA 抑制活性, 其中化合物 $\mathbf{1 b}$ 和 $\mathbf{1 c}$ 的活性最强, 其 $\mathrm{IC}_{50}$ 值 分别为(6.86 2.08$)$ 和 $(9.1 \pm 1.56) \mu \mathrm{g} / \mathrm{mL}$. 此外, 化合物 1a、1d、1e、1g 和 $1 \mathrm{i}$ 的 NA 抑制活性较化合物 $\mathbf{E}$ 和 $\mathbf{F}$ 都有所增强, 说明在 1,2,4-三唑环的硫酮部分引入基团 有助于提高活性.

对于化合物 $1 \mathrm{a} \sim 1 \mathbf{k}$, 当 $\mathrm{R}$ 为 $\mathrm{C}_{2} \mathrm{H}_{5}$ (1b)时, $\mathrm{NA}$ 抑制 活性最佳, 其 $\mathrm{IC}_{50}$ 值为 $(6.86 \pm 2.08) \mu \mathrm{g} / \mathrm{mL}$; 当 $\mathrm{R}$ 为 $\mathrm{C}_{3} \mathrm{H}_{7}$ (1c)时, NA 抑制活性次之, 其 $\mathrm{IC}_{50}$ 值为 $(9.1 \pm 1.56) \mu \mathrm{g} /$ $\mathrm{mL}$; 当 $\mathrm{R}$ 替换成 $\mathrm{CH}_{3}(\mathbf{1 a}) 、 n-\mathrm{C}_{4} \mathrm{H}_{9}(\mathbf{1 e})$ 和 $n-\mathrm{C}_{5} \mathrm{H}_{11}(\mathbf{1 g})$ 时, NA 抑制活性较化合物 $1 b$ 和 $1 \mathrm{c}$ 略有降低, 当 $\mathrm{R}$ 替换 成 4- $\mathrm{CNC}_{6} \mathrm{H}_{4} \mathrm{CH}_{2}$ (1k) 时, $\mathrm{NA}$ 抑制活性较化合物 $\mathbf{1 b}$ 和 $\mathbf{1 c}$
大大降低，其 $\mathrm{IC}_{50}$ 值为 $(20.15 \pm 1.1) \mu \mathrm{g} / \mathrm{mL}$ ，当 $\mathrm{R}$ 为 $4-\mathrm{NO}_{2} \mathrm{C}_{6} \mathrm{H}_{4} \mathrm{CH}_{2}(\mathbf{1 j})$ 时, $\mathrm{NA}$ 抑制率仅为 $(45 \pm 7.57) \%$, 说 明 $\mathrm{R}$ 部位更加适合引入小基团 $\left(\mathrm{C}_{2} \mathrm{H}_{5}, \mathrm{C}_{3} \mathrm{H}_{7}\right)$, 有利于增 加与 NA 活性位点的相互作用, 提高 NA 抑制活性, 缩 短或延长碳链均不利于提高活性; 引入芳基, NA 抑制 活性降低; 化合物 1d 与 1c、1f 与 $1 \mathrm{e} 、 1 \mathrm{~h}$ 与 $1 \mathrm{~g}$ 相比 NA 抑制活性均有所下降, 说明取代基支链化不利于提高 $\mathrm{NA}$ 抑制活性; 把 $\mathrm{R}$ 由疏水基团 $\mathrm{C}_{2} \mathrm{H}_{5}$ (1b)换成极性基团 $\mathrm{CH}_{2} \mathrm{COOH}$ (1i), 化合物 $\mathbf{1 i}$ 的 $\mathrm{NA}$ 抑制率为(81.07士

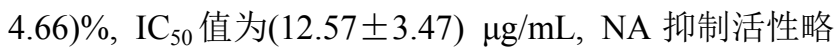
有降低. $\mathrm{R}$ 部位取代基与 NA 抑制活性的关系可归纳为: $\mathrm{C}_{2} \mathrm{H}_{5}>\mathrm{C}_{3} \mathrm{H}_{7}>\mathrm{CH}_{3}, n-\mathrm{C}_{4} \mathrm{H}_{9}>\mathrm{CH}_{2} \mathrm{COOH}>n-\mathrm{C}_{5} \mathrm{H}_{11}>$ $i-\mathrm{Pr}>\mathrm{CH}\left(\mathrm{CH}_{2} \mathrm{CH}_{3}\right)_{2}>\sec -\mathrm{C}_{4} \mathrm{H}_{9}>4-\mathrm{NCC}_{6} \mathrm{H}_{4} \mathrm{CH}_{2}>4-$ $\mathrm{O}_{2} \mathrm{NC}_{6} \mathrm{H}_{4} \mathrm{CH}_{2}$.

将化合物 $\mathbf{1 b}$ 和 $\mathbf{1 c}$ 的 $\mathrm{N}=\mathrm{C}$ 还原成 $\mathrm{NHCH}$ 得到 $2 \mathbf{b}$

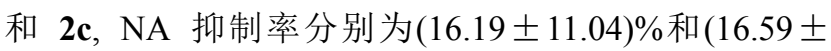
$10.71) \%(40 \mu \mathrm{g} / \mathrm{mL})$, 几乎没有 $\mathrm{NA}$ 抑制活性，说明增大 分子的柔性，可能不利于提高 NA 抑制活性.

表 2 化合物 $\mathbf{1 a} \sim \mathbf{1 k}$ 和 $\mathbf{2 b} \sim \mathbf{2 c}$ 的神经氨酸酶(H1N1)抑制活性 Table 2 Neuraminidase (H1N1) inhibitory activities of compounds $1 \mathrm{a} \sim 1 \mathrm{k}$ and $\mathbf{2 b} \sim \mathbf{2 c}$

\begin{tabular}{|c|c|c|c|}
\hline Compd. & $\mathrm{R}$ & $\begin{array}{l}\text { Inhibition rate/ } \\
\%(40 \mu \mathrm{g} / \mathrm{mL})\end{array}$ & $\mathrm{IC}_{50} /\left(\mu \mathrm{g} \cdot \mathrm{mL}^{-1}\right)$ \\
\hline $1 \mathbf{a}$ & $\mathrm{CH}_{3}$ & $76.11 \pm 2.41$ & $10.6 \pm 2.58$ \\
\hline $1 b$ & $\mathrm{C}_{2} \mathrm{H}_{5}$ & $79.11 \pm 0.64$ & $6.86 \pm 2.08$ \\
\hline $1 \mathrm{c}$ & $n-\operatorname{Pr}$ & $84.05 \pm 1.03$ & $9.10 \pm 1.56$ \\
\hline 1d & $i-\operatorname{Pr}$ & $74.34 \pm 1.39$ & $14.58 \pm 2.54$ \\
\hline $1 \mathrm{e}$ & $n-\mathrm{C}_{4} \mathrm{H}_{9}$ & $78.03 \pm 5.48$ & $10.81 \pm 5.14$ \\
\hline 1f & sec $-\mathrm{C}_{4} \mathrm{H}_{9}$ & $57.47 \pm 8.95$ & $28.2 \pm 11.26$ \\
\hline $1 g$ & $n-\mathrm{C}_{5} \mathrm{H}_{11}$ & $74.48 \pm 4.96$ & $13.89 \pm 4.82$ \\
\hline $1 \mathrm{~h}$ & $\mathrm{CH}\left(\mathrm{CH}_{2} \mathrm{CH}_{3}\right)_{2}$ & $73.01 \pm 1.19$ & $20.12 \pm 1.55$ \\
\hline $1 \mathbf{i}$ & $\mathrm{CH}_{2} \mathrm{COOH}$ & $81.07 \pm 4.66$ & $12.57 \pm 3.47$ \\
\hline $\mathbf{1 j}$ & $4-\mathrm{O}_{2} \mathrm{NC}_{6} \mathrm{H}_{4} \mathrm{CH}_{2}$ & $45.00 \pm 7.57$ & - \\
\hline $1 k$ & $4-\mathrm{NCC}_{6} \mathrm{H}_{4} \mathrm{CH}_{2}$ & $67.37 \pm 1.43$ & $20.15 \pm 1.1$ \\
\hline $2 b$ & $\mathrm{C}_{2} \mathrm{H}_{5}$ & $16.19 \pm 11.04$ & - \\
\hline $2 c$ & $n-\operatorname{Pr}$ & $16.59 \pm 10.71$ & - \\
\hline $\mathbf{E}$ & - & $75.81 \pm 2.24$ & $14.97 \pm 0.70$ \\
\hline $\mathbf{F}$ & - & $79.79 \pm 0.71$ & $14.68 \pm 0.49$ \\
\hline Zanamivir & - & $87.54 \pm 3.40^{a}$ & $0.000047 \pm 0.000007$ \\
\hline
\end{tabular}

${ }^{a}$ The concentration is $0.004 \mu \mathrm{g} / \mathrm{mL}$.

\section{4 分子对接}

为深入研究活性化合物与 NA 的相互作用情况, 将 $\mathrm{NA}$ 抑制活性最佳的化合物 $\mathbf{1 b}$ 和 $\mathbf{1 b}$ 的 $\mathrm{N}=\mathrm{C}$ 还原产物 2b 与 NA (PDA entry: 3TI6)的活性位点进行分子对接, 并与化合物 $\mathbf{E}$ 与 NA (PDA entry: 3TI6)的活性位点分子 对接结果进行对比分析.

如图 $5 \mathrm{a}$ 和 $5 \mathrm{~b}$ 所示, 化合物 $\mathbf{1 b}$ 的 4-OH-3-OCH 3 $\operatorname{Arg} 118, \operatorname{Arg} 292$ 和 $\operatorname{Arg} 371$ (site 1)形成的氢键作用力较 化合物 $\mathbf{E}$ 有所增强, 化合物 $\mathbf{1 b}$ 的 1,2,4-三唑环上的氮原 
子以及碳氮双键的氮原子能与 Arg152 和 Asp151 (site 2) 形成氢键相互作用, 另外, 化合物 $\mathbf{1 b}$ 的 $\mathrm{SC}_{2} \mathrm{H}_{5}$ 如同奥司 他韦羧酸的异戊基一样具有疏水作用. 化合物 $\mathbf{1 b}$ 的对 接结果与化合物 $\mathbf{E}$ 的相比较, 位点作用力增强, 同时引 入的硫醚基团还产生了疏水作用, 最终表现出更优的抑 制活性.

由于将 “ $\mathrm{N}=\mathrm{C}$ ” 还原成 “ $\mathrm{NH}-\mathrm{CH}$ ” 导致化合物 $2 \mathbf{b}$ 的构象发生变化, 如图 $5 \mathrm{c}$ 和 $5 \mathrm{~d}$ 所示, 化合物 $\mathbf{2 b}$ 的 4-OH-3- $\mathrm{OCH}_{3}$ 与 $\mathrm{Arg} 118, \mathrm{Arg} 292$ 和 $\mathrm{Arg} 371$ (site 1)相隔 距离加长, 氢键作用消失, 与 Ser179, Arg152 形成氢键 相互作用, 但它们都不是 NA 活性位点, 化合物 $\mathbf{2 b}$ 与奥 司他韦羧酸在 NA 活性位点的堆叠(图 5d)也显示了化合 物 $\mathbf{2 b}$ 的 4-OH-3- $-\mathrm{OCH}_{3}$ 与奥司他韦羧酸的羧基的重叠度 很低. 化合物 $\mathbf{2 b}$ 的 1,2,4-三唑环上的氮原子以及碳氮双 键的氮原子与 Asp151 (site 2)和 Try406 形成氢键相互作 用, 其中, 只有 Asp151 (site 2)为 NA 活性位点, 同时, 化合物 $\mathbf{2 b}$ 的 $\mathrm{SC}_{2} \mathrm{H}_{5}$ 与奥司他韦羧酸的异戊基重叠度也 很低. 这些可能是化合物 $2 \mathbf{b}$ 的 NA 抑制活性大大降低的 原因.

\section{2 结论}

设计并合成了 13 种 1,2,4-三唑-3-硫醚衍生物(1) $\mathbf{1 k}$ 和 $2 \mathbf{b} \sim 2 \mathbf{c}$ ), 其化学结构经 ${ }^{1} \mathrm{H} N \mathrm{NMR},{ }^{13} \mathrm{C} \mathrm{NMR}, \mathrm{MS}$ 和 元素分析等方法确证. 通过单晶 $\mathrm{X}$ 射线衍射进一步确定 化合物 $1 \mathrm{c}$ 的分子为硫醚结构, $\mathrm{N}=\mathrm{C}$ 键为 $E$ 式构型. NA
(H1N1)的抑制活性表明，化合物 1 具有较好的 NA 抑制 活性，其中化合物 $\mathbf{1 b}$ 和 $1 \mathrm{c}$ 的 $\mathrm{NA}$ 抑制活性最佳，其 $\mathrm{IC}_{50}$ 值分别为(6.86 \pm 2.08$)$ 和 $(9.1 \pm 1.56) \mu \mathrm{g} / \mathrm{mL}$. 构效关系分 析发现, 硫上引入小的直链烷基有利于提高活性, 将 $\mathrm{N}$ $=\mathrm{C}$ 还原成 $\mathrm{NH}-\mathrm{CH}$ 后 $\mathrm{NA}$ 抑制活性大大降低; 同时通 过分子对接分析了活性化合物与 NA 的可能作用模式.

\section{3 实验部分}

\section{1 仪器与试剂}

ZF-2 型三用紫外仪 (上海安亭电子仪器厂); R$1002 \mathrm{~N}$ 型旋转蒸发仪、SHB-III型循环水式多用真空洜 (郑州长城科工贸有限公司); RY-1G 型熔点仪(天津天光 光学仪器有限公司); VARIANINOVA-400 核磁共振仪 (美国 Varian 公司), ${ }^{1} \mathrm{H}$ NMR 共振频率为 $400 \mathrm{MHz},{ }^{13} \mathrm{C}$ NMR 共振频率为 $100 \mathrm{MHz}, \mathrm{TMS}$ 为内标; Bruker AXS SMART 1000 CCDX 射线单晶衍射仪(德国); Agilent 1100 Series 型高效液相色谱-质谱联用系统(EI, 美国 Agilent 公司); VARIO EL III 元素分析仪(德国).

薄层层析硅胶板(烟台江友硅胶开发有限公司). 溶 剂和试剂均为市售分析纯或化学纯.

\section{2 实验方法}

\subsection{1 目标化合物 $\mathbf{1 a} \sim \mathbf{1 k}$ 的合成}

将 $1.0 \mathrm{mmol}$ 化合物 $\mathbf{E} 、 3 \mathrm{mmol}$ 氢氧化钾和 $10 \mathrm{~mL}$ 水混合均匀, $0 \sim 5{ }^{\circ} \mathrm{C}$ 下, 缓慢滴加 $2.24 \mathrm{mmol}$ 硫酸二甲
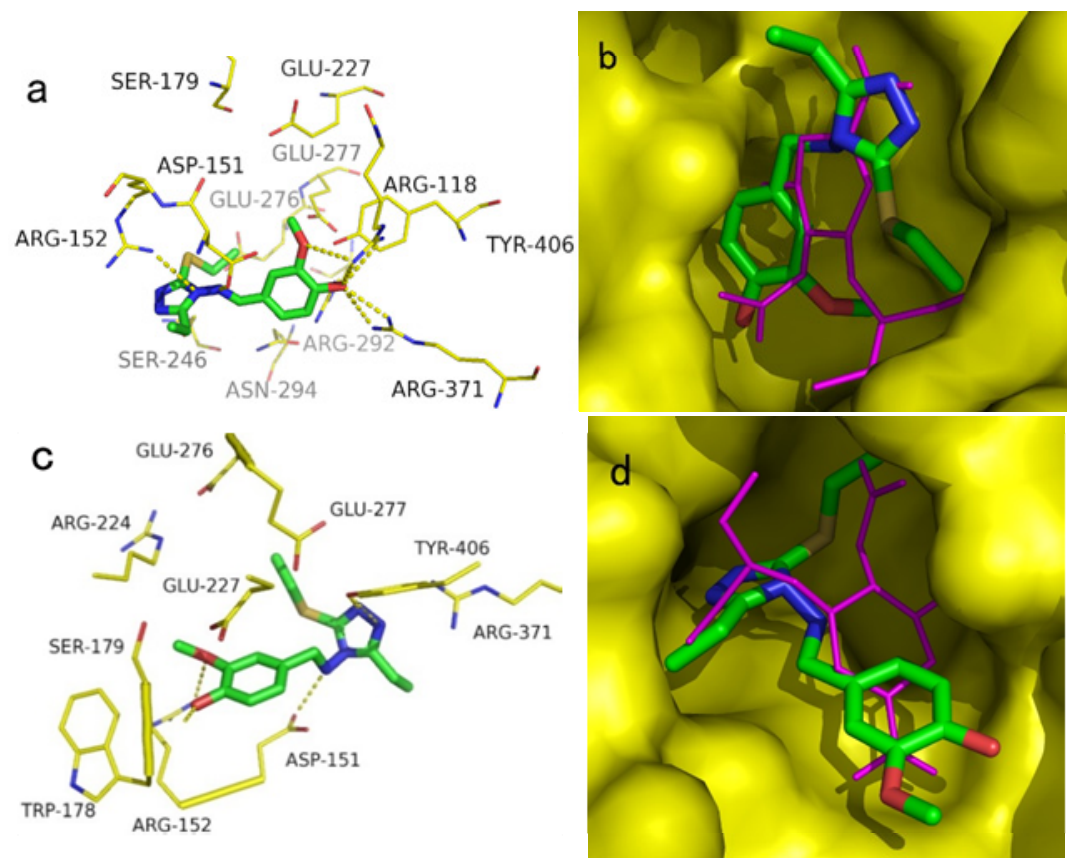

图 5 化合物 $\mathbf{1 b}$ 和 $\mathbf{2 b}$ 与 A/H1N1-NA (3TI6)的分子对接模型

Figure 5 Interaction models of $\mathbf{1 b}$ and $\mathbf{2 b}$ with A/H1N1-NA (3TI6)

(a, c) Binding models of $\mathbf{1 b}$ (a) and $\mathbf{2 b}$ (c) with A/H1N1-NA. The H-bond interactions are depicted as yellow dashed lines. (b, d) Comparison of the interactions of $\mathbf{1 b}$ (b) and 2b (d) and oseltamivir acid with A/H1N1-NA by structural superposition. 
酯的乙醇 $(10 \mathrm{~mL})$ 溶液, 滴毕, 室温搅拌 $1 \mathrm{~h}$ [薄层色谱 (TLC) 跟踪], 析出固体, 抽滤、水洗、乙酸乙酯重结晶 得化合物 1a. 将 $1.0 \mathrm{mmol}$ 化合物 $\mathbf{E} 、 2.0 \mathrm{mmol}$ 溴代烷 烃和 $0.6 \mathrm{mmol}$ 碳酸钾溶于 $4 \mathrm{~mL}$ 乙醇, 回流反应 $13.5 \mathrm{~h}$ (TLC 跟踪), 冷却、减压除去溶剂, 加入乙酸乙酯, 饱和 食盐水洗涤, 有机层用无水硫酸钠干燥、过滤、减压除 去乙酸乙酯, 加入二氯甲烷/石油醚 $(V: V=1: 2)$ 混合 溶剂, 静置, 析出固体, 用乙酸乙酯或乙醇重结晶得化 合物 $\mathbf{1 b} \sim \mathbf{1 h}$. 将 $1.0 \mathrm{mmol}$ 化合物 $\mathbf{E}$ 和 $1.0 \mathrm{mmol}$ 氯乙酸 溶于含有 $0.15 \mathrm{~mL}$ 吡啶的 $6 \mathrm{~mL}$ 乙醇混合溶液中, 回流反 应 $6 \mathrm{~h}$ (TLC 跟踪), 冷却、减压除去溶剂得黄色粘稠状液 体, 加入 $3 \mathrm{~mL}$ 水, 析出黄色晶体, 抽滤、乙酸乙酯重结 晶得化合物 1i. $1.0 \mathrm{mmol}$ 化合物 $\mathbf{E}$, 将 $1.1 \mathrm{mmol} 4$-取代 芐溴, $5 \mathrm{~mL}$ 乙醇, 10 滴三乙胺, 回流反应 $1.5 \sim 2.5 \mathrm{~h}$ (TLC 跟踪), 冷却、减压除去溶剂, 析出固体, 乙醇洗涤, 乙酸乙酯重结晶得化合物 $\mathbf{1 j}$ 和 $\mathbf{1 k}$.

(E)-4-(4-差基-3-甲氧基苯基亚甲氨基)-5-乙基- $4 \mathrm{H}$ 1,2,4-三唑-3-甲硫醚(1a): 白色固体, 收率 39.7\%. m.p. $178 \sim 180{ }^{\circ} \mathrm{C} ;{ }^{1} \mathrm{H}$ NMR $\left(400 \mathrm{MHz}, \mathrm{DMSO}-d_{6}\right) \delta: 1.25(\mathrm{t}$, $\left.J=7.5 \mathrm{~Hz}, 3 \mathrm{H}, \mathrm{CH}_{3}\right), 2.61\left(\mathrm{~s}, 3 \mathrm{H}, \mathrm{CH}_{3}\right), 2.77$ (t, $J=7.5$ $\left.\mathrm{Hz}, 2 \mathrm{H}, \mathrm{CH}_{2}\right), 3.87$ (s, 3H, OCH 3$), 6.97$ (d, $J=8.2 \mathrm{~Hz}, 1 \mathrm{H}$, $\mathrm{C}_{6} \mathrm{H}_{3}$ ), 7.40 (dd, $\left.J=8.2,1.6 \mathrm{~Hz}, 1 \mathrm{H}, \mathrm{C}_{6} \mathrm{H}_{3}\right), 7.52$ (d, $J=1.6$ $\left.\mathrm{Hz}, 1 \mathrm{H}, \mathrm{C}_{6} \mathrm{H}_{3}\right), 8.68(\mathrm{~s}, 1 \mathrm{H}, \mathrm{NCH}), 10.14(\mathrm{~s}, 1 \mathrm{H}, \mathrm{OH}) ;{ }^{13} \mathrm{C}$ NMR $\left(100 \mathrm{MHz}, \mathrm{DMSO}-d_{6}\right) \delta: 166.9,153.9,152.3,148.7$, 147.2, 125.2, 123.4, 116.2, 111.2, 56.1, 18.6, 15.2, 11.4; MS (ESI) $m / z: 290.83[\mathrm{M}-\mathrm{H}]^{-}$. Anal. calcd for $\mathrm{C}_{13} \mathrm{H}_{16} \mathrm{~N}_{4}-$ $\mathrm{O}_{2} \mathrm{~S}$ : C 53.41, H 5.52, N 19.16; found C 53.45, H 5.46, N 19.14 .

(E)-4-(4-羟基-3-甲氧基苯基亚甲氨基)-5-乙基4H-1,2,4-三唑-3-乙硫醚(1b): 白色固体, 收率 78.7\%. m.p. $137 \sim 139{ }^{\circ} \mathrm{C} ;{ }^{1} \mathrm{H}$ NMR (400 MHz, DMSO- $d_{6}$ ) $\delta$ : 1.27 (t, $\left.J=7.4 \mathrm{~Hz}, 3 \mathrm{H}, \mathrm{CH}_{3}\right), 1.33$ (t, $J=7.4 \mathrm{~Hz}, 3 \mathrm{H}, \mathrm{CH}_{3}$ ), 2.79 (q, $\left.J=7.4 \mathrm{~Hz}, 2 \mathrm{H}, \mathrm{CH}_{2}\right), 3.14(\mathrm{q}, J=7.4 \mathrm{~Hz}, 2 \mathrm{H}$, $\mathrm{CH}_{2}$ ), 3.89 (s, $\left.3 \mathrm{H}, \mathrm{OCH}_{3}\right), 6.99$ (d, $J=8.0 \mathrm{~Hz}, 1 \mathrm{H}, \mathrm{C}_{6} \mathrm{H}_{3}$ ), $7.42\left(\mathrm{~d}, J=8.0 \mathrm{~Hz}, 1 \mathrm{H}, \mathrm{C}_{6} \mathrm{H}_{3}\right), 7.54\left(\mathrm{~s}, 1 \mathrm{H}, \mathrm{C}_{6} \mathrm{H}_{3}\right), 8.68$ (s, $1 \mathrm{H}, \mathrm{CH}), 10.19(\mathrm{~s}, 1 \mathrm{H}, \mathrm{OH}) ;{ }^{13} \mathrm{C}$ NMR $(100 \mathrm{MHz}$, DMSO- $\left.d_{6}\right) \delta: 167.2,153.9,152.3,148.7,145.9,125.3$, 123.5, 116.2, 111.2, 56.1, 27.3, 18.7, 15.3, 11.3; MS (ESI) $m / z: 305.11[\mathrm{M}-\mathrm{H}]^{-}$. Anal. calcd for $\mathrm{C}_{14} \mathrm{H}_{18} \mathrm{~N}_{4} \mathrm{O}_{2} \mathrm{~S}$ : C 54.88, H 5.92, N 18.29; found C 54.85, H 5.90, N 18.25.

(E)-4-(4-羟基-3-甲氧基苯基亚甲氨基)-5-乙基4H-1,2,4-三唑-3-丙硫醚(1c): 白色固体, 收率 76.4\%. m.p. $119 \sim 121{ }^{\circ} \mathrm{C} ;{ }^{1} \mathrm{H}$ NMR (400 MHz, $\left.\mathrm{CDCl}_{3}\right) \delta: 1.00$ (t, $\left.J=7.3 \mathrm{~Hz}, 3 \mathrm{H}, \mathrm{CH}_{3}\right), 1.35\left(\mathrm{t}, J=7.5 \mathrm{~Hz}, 3 \mathrm{H}, \mathrm{CH}_{3}\right), 1.70 \sim$ $1.79\left(\mathrm{~m}, 2 \mathrm{H}, \mathrm{CH}_{2}\right), 2.86\left(\mathrm{q}, J=7.5 \mathrm{~Hz}, 2 \mathrm{H}, \mathrm{CH}_{2}\right), 3.19$ (t,
$\left.J=7.2 \mathrm{~Hz}, 2 \mathrm{H}, \mathrm{SCH}_{2}\right), 4.00\left(\mathrm{~s}, 3 \mathrm{H}, \mathrm{OCH}_{3}\right), 6.84(\mathrm{~s}, 1 \mathrm{H}$, $\mathrm{OH}), 7.06\left(\mathrm{~d}, J=8.4 \mathrm{~Hz}, 1 \mathrm{H}, \mathrm{C}_{6} \mathrm{H}_{3}\right), 7.28(\mathrm{~d}, J=8.4 \mathrm{~Hz}$, $\left.1 \mathrm{H}, \mathrm{C}_{6} \mathrm{H}_{3}\right), 7.49\left(\mathrm{~s}, 1 \mathrm{H}, \mathrm{C}_{6} \mathrm{H}_{3}\right), 8.41(\mathrm{~s}, 1 \mathrm{H}, \mathrm{CH}) ;{ }^{13} \mathrm{C} \mathrm{NMR}$ $\left(100 \mathrm{MHz}, \mathrm{CDCl}_{3}\right) \delta: 163.9,155.1,150.7,147.4,146.1$, 125.7, 124.1, 114.7, 108.7, 56.2, 35.5, 22.8, 18.9, 13.2, 11.2; MS (ESI) $m / z$ : $318.88[\mathrm{M}-\mathrm{H}]^{-}$. Anal. calcd for $\mathrm{C}_{15} \mathrm{H}_{20} \mathrm{~N}_{4} \mathrm{O}_{2} \mathrm{~S}$ : C 56.23, H 6.29, N 17.49; found C 56.15, H 6.24, N 17.46.

(E)-4-(4-羟基-3-甲氧基苯基亚甲氨基)-5-乙基4H-1,2,4-三唑-3-异丙硫醚 (1d): 淡黄色晶体, 收率 50.0\%. m.p. $170 \sim 171{ }^{\circ} \mathrm{C} ;{ }^{1} \mathrm{H}$ NMR (400 MHz, DMSO$\left.d_{6}\right) \delta: 10.17(\mathrm{~s}, 1 \mathrm{H}, \mathrm{OH}), 8.63(\mathrm{~s}, 1 \mathrm{H}, \mathrm{NCH}), 7.50(\mathrm{~s}, 1 \mathrm{H}$, $\left.\mathrm{C}_{6} \mathrm{H}_{3}\right), 7.39$ (d, $\left.J=8.0 \mathrm{~Hz}, 1 \mathrm{H}, \mathrm{C}_{6} \mathrm{H}_{3}\right), 6.96(\mathrm{~d}, J=8.0 \mathrm{~Hz}$, $\left.1 \mathrm{H}, \mathrm{C}_{6} \mathrm{H}_{3}\right), 3.86\left(\mathrm{~s}, 3 \mathrm{H}, \mathrm{OCH}_{3}\right), 3.68 \sim 3.63(\mathrm{~m}, 1 \mathrm{H}, \mathrm{CH})$, 2.77 (q, $\left.J=7.6 \mathrm{~Hz}, 2 \mathrm{H}, \mathrm{CH}_{2}\right), 1.31$ (d, $J=6.8 \mathrm{~Hz}, 6 \mathrm{H}, 2 \times$ $\left.\mathrm{CH}_{3}\right), 1.25\left(\mathrm{t}, J=7.6 \mathrm{~Hz}, 3 \mathrm{H}, \mathrm{CH}_{3}\right) ;{ }^{13} \mathrm{C}$ NMR $(101 \mathrm{MHz}$, DMSO- $\left.d_{6}\right) \delta$ : 169.9, 166.8, 153.9, 152.4, 148.6, 145.8, 125.3, 123.4, 116.2, 111.2, 56.1, 34.9, 18.7, 11.3; MS (ESI) $m / z: 318.88[\mathrm{M}-\mathrm{H}]^{-}$. Anal. calcd for $\mathrm{C}_{15} \mathrm{H}_{20}-\mathrm{N}_{4} \mathrm{O}_{2} \mathrm{~S}: \mathrm{C}$ 56.23, H 6.29, N 17.49; found C 56.15, H 6.24, N 17.46.

(E)-4-(4-羟基-3- 甲氧基苯基亚甲氨基)-5-乙基4H-1,2,4-三唑-3-丁硫醚(1e): 浅黄色固体, 收率 75.7\%. m.p. $103 \sim 106{ }^{\circ} \mathrm{C} ;{ }^{1} \mathrm{H}$ NMR (400 MHz, DMSO- $\left.d_{6}\right) \delta$ : $0.88\left(\mathrm{t}, J=7.4 \mathrm{~Hz}, 3 \mathrm{H}, \mathrm{CH}_{3}\right), 1.25\left(\mathrm{t}, J=7.5 \mathrm{~Hz}, 3 \mathrm{H}, \mathrm{CH}_{3}\right)$, $1.34 \sim 1.43\left(\mathrm{~m}, 2 \mathrm{H}, \mathrm{C}_{4} \mathrm{H}_{7}-\mathrm{CH}_{2}\right), 1.59 \sim 1.66\left(\mathrm{~m}, 2 \mathrm{H}, \mathrm{C}_{4} \mathrm{H}_{7}-\right.$ $\mathrm{CH}_{2}$ ), 2.77 (q, $J=7.5 \mathrm{~Hz}, 2 \mathrm{H}, \mathrm{C}_{2} \mathrm{H}_{5}-\mathrm{CH}_{2}$ ), 3.11 (t, $J=7.2$ $\mathrm{Hz}, 2 \mathrm{H}, \mathrm{C}_{4} \mathrm{H}_{7}-\mathrm{CH}_{2}$ ), 3.87 (s, $\left.3 \mathrm{H}, \mathrm{OCH}_{3}\right), 6.97$ (d, $J=8.2$ $\left.\mathrm{Hz}, 1 \mathrm{H}, \mathrm{C}_{6} \mathrm{H}_{3}\right), 7.40\left(\mathrm{dd}, J=8.2,1.6 \mathrm{~Hz}, 1 \mathrm{H}, \mathrm{C}_{6} \mathrm{H}_{3}\right), 7.52$ $\left(\mathrm{d}, J=1.6 \mathrm{~Hz}, 1 \mathrm{H}, \mathrm{C}_{6} \mathrm{H}_{3}\right), 8.66(\mathrm{~s}, 1 \mathrm{H}, \mathrm{N}=\mathrm{CH}), 10.15(\mathrm{~s}$, $1 \mathrm{H}, \mathrm{OH}) ;{ }^{13} \mathrm{C}$ NMR $\left(100 \mathrm{MHz}\right.$, DMSO- $\left.d_{6}\right) \delta: 167.3,153.9$, 152.3, 148.6, 146.0, 125.3, 123.5,1 16.2, 111.2, 56.1, 32.7, 31.5, 21.5, 18.7, 13.8, 11.3; MS (ESI) $m / z: 332.92$ [M$\mathrm{H}]^{-}$. Anal. calcd for $\mathrm{C}_{16} \mathrm{H}_{22} \mathrm{~N}_{4} \mathrm{O}_{2} \mathrm{~S}$ : C 57.46, H 6.63, N 16.75; found C 57.44, H 6.71, N 16.72.

(E)-4-(4-羟基-3-甲氧基苯基亚甲氨基)-5-乙基4H-1,2,4-三唑-3-仲丁硫醚(1f): 白色固体, 收率 52.5\%. m.p. $183 \sim 186{ }^{\circ} \mathrm{C} ;{ }^{1} \mathrm{H}$ NMR (400 MHz, DMSO- $\left.d_{6}\right) \delta$ : $0.93\left(\mathrm{t}, J=7.4 \mathrm{~Hz}, 3 \mathrm{H}, \mathrm{CH}_{3}\right), 1.25\left(\mathrm{t}, J=7.5 \mathrm{~Hz}, 3 \mathrm{H}, \mathrm{CH}_{3}\right)$, $1.29\left(\mathrm{~d}, J=6.8 \mathrm{~Hz}, 3 \mathrm{H}, \mathrm{CH}_{3}\right), 1.55 \sim 1.66\left(\mathrm{~m}, 2 \mathrm{H}, \mathrm{CH}_{2}\right)$, $2.76\left(\mathrm{q}, J=7.5 \mathrm{~Hz}, 2 \mathrm{H}, \mathrm{CH}_{2}\right), 3.45 \sim 3.50(\mathrm{~m}, 1 \mathrm{H}, \mathrm{CH})$, $3.87\left(\mathrm{~s}, 3 \mathrm{H}, \mathrm{OCH}_{3}\right), 6.97\left(\mathrm{~d}, J=8.2 \mathrm{~Hz}, 1 \mathrm{H}, \mathrm{C}_{6} \mathrm{H}_{3}\right), 7.40$ (dd, $\left.J=8.2,1.6 \mathrm{~Hz}, 1 \mathrm{H}, \mathrm{C}_{6} \mathrm{H}_{3}\right), 7.52(\mathrm{~d}, J=1.6 \mathrm{~Hz}, 1 \mathrm{H}$, $\left.\mathrm{C}_{6} \mathrm{H}_{3}\right), 8.65(\mathrm{~s}, 1 \mathrm{H}, \mathrm{N}=\mathrm{CH}), 10.14(\mathrm{~s}, 1 \mathrm{H}, \mathrm{OH}) ;{ }^{13} \mathrm{C} \mathrm{NMR}$ $\left(100 \mathrm{MHz}\right.$, DMSO- $\left.d_{6}\right) \delta: 167.8,153.9,152.3,148.6,145.0$, $125.3,123.5,116.2,111.2,56.1,45.9,29.5,21.2,18.7$, 
11.6, 11.3; MS (ESI) $m / z$ : $332.92[\mathrm{M}-\mathrm{H}]^{-}$. Anal. calcd for $\mathrm{C}_{16} \mathrm{H}_{22} \mathrm{~N}_{4} \mathrm{O}_{2} \mathrm{~S}$ : C 57.46, H 6.63, N 16.75; found C 57.44, H 6.71, N 16.72 .

(E)-4-(4-羟基-3-甲氧基苯基亚甲氨基)-5-乙基- $4 \mathrm{H}$ 1,2,4-三唑-3-戊硫醚(1g): 白色固体, 收率 75.2\%. m.p. $114 \sim 116{ }^{\circ} \mathrm{C} ;{ }^{1} \mathrm{H}$ NMR (400 MHz, DMSO- $\left.d_{6}\right) \delta: 0.85$ (t, $\left.J=7.1 \mathrm{~Hz}, 3 \mathrm{H}, \mathrm{CH}_{3}\right), 1.25\left(\mathrm{t}, J=7.5 \mathrm{~Hz}, 3 \mathrm{H}, \mathrm{CH}_{3}\right), 1.38 \sim$ $1.27\left(\mathrm{~m}, 4 \mathrm{H}, \mathrm{CH}_{2}\right), 1.68 \sim 1.59\left(\mathrm{~m}, 2 \mathrm{H}, \mathrm{CH}_{2}\right), 2.76(\mathrm{q}, J=$ $7.5 \mathrm{~Hz}, 2 \mathrm{H}, \mathrm{C}_{2} \mathrm{H}_{5}-\mathrm{CH}_{2}$ ), 3.09 (t, J=7.2 Hz, $2 \mathrm{H}, \mathrm{C}_{5} \mathrm{H}_{11^{-}}$ $\left.\mathrm{CH}_{2}\right), 3.87\left(\mathrm{~s}, 3 \mathrm{H}, \mathrm{OCH}_{3}\right), 6.97\left(\mathrm{~d}, J=8.2 \mathrm{~Hz}, 1 \mathrm{H}, \mathrm{C}_{6} \mathrm{H}_{3}\right)$, $7.40\left(\mathrm{dd}, J=8.2,1.8 \mathrm{~Hz}, 1 \mathrm{H}, \mathrm{C}_{6} \mathrm{H}_{3}\right), 7.52(\mathrm{~d}, J=1.8 \mathrm{~Hz}$, $\left.1 \mathrm{H}, \mathrm{C}_{6} \mathrm{H}_{3}\right), 8.66(\mathrm{~s}, 1 \mathrm{H}, \mathrm{N}=\mathrm{CH}), 10.14(\mathrm{~s}, 1 \mathrm{H}, \mathrm{OH}) ;{ }^{13} \mathrm{C}$ NMR (100 MHz, DMSO- $\left.d_{6}\right) \delta: 167.3,153.9,152.3,148.6$, 146.0, 125.3, 123.5, 116.2, 111.2, 56.1, 33.01, 30.5, 29.1, 22.1, 18.7, 14.2, 11.3; MS (ESI) $m / z: 347.01[\mathrm{M}-\mathrm{H}]^{-}$. Anal. calcd for $\mathrm{C}_{17} \mathrm{H}_{24} \mathrm{~N}_{4} \mathrm{O}_{2} \mathrm{~S}$ : C 58.60, H 6.94, N 16.08; found C 58.68, H 6.91, N 16.05 .

(E)-4-(4-羟基-3-甲氧基苯基亚甲氨基)-5-乙基4H-1,2,4-三唑-3-(戊-3)-硫醚(1h): 白色固体, 收率 48.2\%. m.p. 189 191 ${ }^{\circ} \mathrm{C}$; ${ }^{1} \mathrm{H}$ NMR (400 MHz, DMSO$\left.d_{6}\right) \delta: 0.93\left(\mathrm{t}, J=7.2 \mathrm{~Hz}, 6 \mathrm{H}, \mathrm{CH}_{3}\right), 1.25(\mathrm{t}, J=7.2 \mathrm{~Hz}, 3 \mathrm{H}$, $\left.\mathrm{CH}_{3}\right), 1.55 \sim 1.65\left(\mathrm{~m}, 4 \mathrm{H}, \mathrm{CH}_{2}\right), 2.76(\mathrm{q}, J=7.2 \mathrm{~Hz}, 2 \mathrm{H}$, $\left.\mathrm{CH}_{2}\right), 3.29 \sim 3.32(\mathrm{~m}, 1 \mathrm{H}, \mathrm{CH}), 3.86\left(\mathrm{~s}, 3 \mathrm{H}, \mathrm{OCH}_{3}\right), 6.97$ (d, $\left.J=8.0 \mathrm{~Hz}, 1 \mathrm{H}, \mathrm{C}_{6} \mathrm{H}_{3}\right), 7.40\left(\mathrm{~d}, J=8.0 \mathrm{~Hz}, 1 \mathrm{H}, \mathrm{C}_{6} \mathrm{H}_{3}\right)$, 7.52 (s, $\left.1 \mathrm{H}, \mathrm{C}_{6} \mathrm{H}_{3}\right), 8.67$ (s, $\left.1 \mathrm{H}, \mathrm{NCH}\right), 10.18(\mathrm{~s}, 1 \mathrm{H}, \mathrm{OH})$; ${ }^{13} \mathrm{C}$ NMR (100 MHz, DMSO- $\left.d_{6}\right) \delta: 167.8,153.9,152.3$, 148.6, 145.0, 125.3, 123.5, 116.2, 111.2, 56.1, 53.2, 27.1, 18.7, 11.5, 11.3; MS (ESI) $m / z$ : $347.01[\mathrm{M}-\mathrm{H}]^{-}$. Anal. calcd for $\mathrm{C}_{17} \mathrm{H}_{24} \mathrm{~N}_{4} \mathrm{O}_{2} \mathrm{~S}$ : C 58.60, H 6.94, N 16.08; found C $58.68, \mathrm{H} 6.91, \mathrm{~N} 16.05$.

(E)-2-((5-乙基-4-((4-差基-3-甲氧基亚苠基)氨基)4H-1,2,4-三唑-3-基)硫代)乙酸(1i): 黄色固体, 收率 43.0\%. m.p. $174.5 \sim 176{ }^{\circ} \mathrm{C} ;{ }^{1} \mathrm{H}$ NMR (400 MHz, DMSO$\left.d_{6}\right) \delta: 1.23\left(\mathrm{t}, J=7.6 \mathrm{~Hz}, 3 \mathrm{H}, \mathrm{CH}_{2} \mathrm{CH}_{3}\right), 2.73 \sim 2.78(\mathrm{~m}$, $\left.2 \mathrm{H}, \mathrm{CH}_{2}\right), 3.85\left(\mathrm{~s}, 3 \mathrm{H}, \mathrm{OCH}_{3}\right), 3.99\left(\mathrm{~s}, 2 \mathrm{H}, \mathrm{SCH}_{2}\right), 7.50 \sim$ $6.95\left(\mathrm{~m}, 3 \mathrm{H}, \mathrm{C}_{6} \mathrm{H}_{3}\right), 8.66$ (s, 1H, NCH), $10.13(\mathrm{~s}, 1 \mathrm{H}, \mathrm{OH})$, $12.91(\mathrm{~s}, 1 \mathrm{H}, \mathrm{COOH}) ;{ }^{13} \mathrm{C}$ NMR (101 MHz, DMSO- $\left.d_{6}\right) \delta$ : 167.6, 153. 9, 152.3, 148.6, 145.1, 125.3, 123.5, 116.2, 111.2, 56.1, 39.3, 23.6, 18.7, 11.3; MS (ESI) $m / z: 334.82$ $[\mathrm{M}-\mathrm{H}]^{-}$. Anal. calcd for $\mathrm{C}_{14} \mathrm{H}_{16} \mathrm{~N}_{4} \mathrm{O}_{4} \mathrm{~S}$ : C 49.99, H 4.79, $\mathrm{N} 16.66$; found $\mathrm{C} 50.03, \mathrm{H} 4.85, \mathrm{~N} 16.64$.

(E)-4-(4-羟基-3-甲氧基苯基亚甲氨基)-5-乙基-

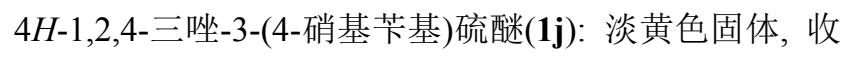
率 67.8\%. m.p. $154 \sim 156{ }^{\circ} \mathrm{C} ;{ }^{1} \mathrm{H}$ NMR $(400 \mathrm{MHz}$, DMSO- $\left.d_{6}\right) \delta: 1.21\left(\mathrm{t}, J=7.4 \mathrm{~Hz}, 3 \mathrm{H}, \mathrm{CH}_{3}\right), 2.73$ (q, $J=7.4$
$\left.\mathrm{Hz}, 2 \mathrm{H}, \mathrm{CH}_{2}\right), 3.85$ (s, 3H, $\mathrm{OCH}_{3}$ ), 4.47 (s, $\left.2 \mathrm{H}, \mathrm{CH}_{2}\right), 6.94$ (d, $\left.J=8.0 \mathrm{~Hz}, 1 \mathrm{H}, \mathrm{C}_{6} \mathrm{H}_{3}\right), 7.33\left(\mathrm{~d}, J=8.0 \mathrm{~Hz}, 1 \mathrm{H}, \mathrm{C}_{6} \mathrm{H}_{3}\right.$ ), $7.42\left(\mathrm{~s}, 1 \mathrm{H}, \mathrm{C}_{6} \mathrm{H}_{3}\right), 7.59$ (d, $\left.J=8.0 \mathrm{~Hz}, 2 \mathrm{H}, \mathrm{C}_{6} \mathrm{H}_{4}\right), 8.15$ (d, $\left.J=8.0 \mathrm{~Hz}, 2 \mathrm{H}, \mathrm{C}_{6} \mathrm{H}_{4}\right), 8.57(\mathrm{~s}, 1 \mathrm{H}, \mathrm{NH}), 10.20(\mathrm{~s}, 1 \mathrm{H}$, $\mathrm{OH}) ;{ }^{13} \mathrm{C}$ NMR (100 MHz, DMSO- $\left.d_{6}\right) \delta: 167.3,154.1$, $152.4,148.6,147.1,146.2,145.1,130.6,125.3,123.9$, 123.3, 116.1, 111.2, 56.1, 36.2, 18.7, 11.2; MS (ESI) $\mathrm{m} / \mathrm{z}$ : $412.11[\mathrm{M}-\mathrm{H}]^{-}$. Anal. calcd for $\mathrm{C}_{19} \mathrm{H}_{19} \mathrm{~N}_{5} \mathrm{O}_{4} \mathrm{~S}: \mathrm{C} 55.20, \mathrm{H}$ 4.63, N 16.94; found C 55.07, H 4.56, N 16.91.

(E)-4-(4-羟基-3-甲氧基苯基亚甲氨基)-5-乙基4H-1,2,4-三唑-3-(4-氭基苄基)硫醚(1k): 白色固体, 收率 $55.9 \%$. m.p. $157 \sim 159{ }^{\circ} \mathrm{C} ;{ }^{1} \mathrm{H}$ NMR (400 MHz, DMSO$\left.d_{6}\right) \delta: 1.23\left(\mathrm{t}, J=7.4 \mathrm{~Hz}, 3 \mathrm{H}, \mathrm{CH}_{3}\right), 2.74(\mathrm{q}, J=7.4 \mathrm{~Hz}, 2 \mathrm{H}$, $\mathrm{CH}_{2}$ ), 3.87 (s, 3H, $\left.\mathrm{OCH}_{3}\right), 4.43$ (s, $\left.2 \mathrm{H}, \mathrm{CH}_{2}\right), 6.96$ (d, $J=$ $\left.8.0 \mathrm{~Hz}, 1 \mathrm{H}, \mathrm{C}_{6} \mathrm{H}_{3}\right), 7.35\left(\mathrm{~d}, J=8.0 \mathrm{~Hz}, 1 \mathrm{H}, \mathrm{C}_{6} \mathrm{H}_{3}\right), 7.44(\mathrm{~s}$, $\left.1 \mathrm{H}, \mathrm{C}_{6} \mathrm{H}_{3}\right), 7.52\left(\mathrm{~d}, J=7.8 \mathrm{~Hz}, 2 \mathrm{H}, \mathrm{C}_{6} \mathrm{H}_{4}\right), 7.77$ (d, $J=7.8$ $\left.\mathrm{Hz}, 2 \mathrm{H}, \mathrm{C}_{6} \mathrm{H}_{4}\right), 8.57$ (s, $\left.1 \mathrm{H}, \mathrm{NCH}\right), 10.17(\mathrm{~s}, 1 \mathrm{H}, \mathrm{OH}) ;{ }^{13} \mathrm{C}$ NMR (100 MHz, DMSO- $\left.d_{6}\right) \delta: 167.2,154.1,152.4,148.6$, $145.2,143.9,132.7,130.4,125.3,123.4,119.2,116.1$, 111.2, 110.5, 56.1, 36.5, 18.7, 11.3; MS (ESI) $\mathrm{m} / z: 392.10$ $[\mathrm{M}-\mathrm{H}]^{-}$. Anal. calcd for $\mathrm{C}_{20} \mathrm{H}_{19} \mathrm{~N}_{5} \mathrm{O}_{2} \mathrm{~S}: \mathrm{C} 61.05, \mathrm{H} 4.87$, N 17.80; found C 61.02, H 4.94, N 17.77.

\subsection{2 目标化合物 $\mathbf{2 b} \sim \mathbf{2} \mathbf{c}$ 的合成}

将 $1.0 \mathrm{mmol} \mathbf{1 b}$ 或 $1 \mathbf{c}, 25 \mathrm{mmol}$ 硼氢化钠溶于 $16 \mathrm{~mL}$ 甲醇溶液, 室温反应 $0.8 \sim 1 \mathrm{~h}$, 减压除去溶剂, 乙酸乙 酯萃取 $(30 \mathrm{~mL} \times 2)$, 饱和食盐水洗涤, 无水硫酸钠干燥, 过滤, 减压除去乙酸乙酯, 重结晶 $[V$ (石油醚) : $V$ (二氯 甲烷 $)=2: 1$ ] 得化合物 $\mathbf{2 b}$ 和 $\mathbf{2 c}$.

(E)-4-(4- 羟基-3- 甲氧基苯基甲氨基)-5-乙基4H-1,2,4-三唑-3-乙硫醚(2b): 白色固体, 收率 $82.0 \%$. m.p. $138 \sim 139{ }^{\circ} \mathrm{C} ;{ }^{1} \mathrm{H}$ NMR (400 MHz, DMSO- $\left.d_{6}\right) \delta$ : $1.11\left(\mathrm{t}, J=7.6 \mathrm{~Hz}, 3 \mathrm{H}, \mathrm{SCH}_{2} \mathrm{CH}_{3}\right), 1.32(\mathrm{t}, J=7.2 \mathrm{~Hz}, 3 \mathrm{H}$, $\left.\mathrm{CH}_{2} \mathrm{CH}_{3}\right), 2.50 \sim 2.42\left(\mathrm{~m}, 2 \mathrm{H}, \mathrm{CH}_{2}\right), 3.15 \sim 3.10(\mathrm{~m}, 2 \mathrm{H}$, $\left.\mathrm{SCH}_{2}\right), 3.72\left(\mathrm{~s}, 3 \mathrm{H}, \mathrm{OCH}_{3}\right), 3.96(\mathrm{~d}, J=4.4 \mathrm{~Hz}, 2 \mathrm{H}$, $\left.\mathrm{NHCH}_{2}\right), 6.71 \sim 6.61\left(\mathrm{~m}, 3 \mathrm{H}, \mathrm{C}_{6} \mathrm{H}_{3}\right), 6.77(\mathrm{~s}, 1 \mathrm{H}, \mathrm{NH})$, $8.96(\mathrm{~s}, 1 \mathrm{H}, \mathrm{OH}) ;{ }^{13} \mathrm{C}$ NMR (101 MHz, DMSO- $\left.d_{6}\right) \delta$ : $157.1,150.4,147.8,146.7,127.6,122.3,115.7,113.9$, 56.0, 54.7, 26.5, 17.7, 15.4, 11.5; MS (ESI) $\mathrm{m} / z$ : 307.06 $[\mathrm{M}-\mathrm{H}]^{-}$. Anal. calcd for $\mathrm{C}_{14} \mathrm{H}_{20} \mathrm{~N}_{4} \mathrm{O}_{2} \mathrm{~S}$ : C 54.52, H 6.54, $\mathrm{N}$ 18.17; found C 54.54, H 6.56, N 18.14 .

(E)-4-(4-差基-3-甲氧基苯基甲氨基)-5-乙基-4H1,2,4-三唑-3-丙硫醚(2c): 白色晶体，收率 $81.0 \%$. m.p. $139 \sim 142{ }^{\circ} \mathrm{C} ;{ }^{1} \mathrm{H}$ NMR (400 MHz, DMSO- $\left.d_{6}\right) \delta: 1.00$ (t, $\left.J=7.6 \mathrm{~Hz}, 3 \mathrm{H}, \mathrm{SCH}_{2} \mathrm{CH}_{2} \mathrm{CH}_{3}\right), 1.14(\mathrm{t}, J=7.6 \mathrm{~Hz}, 3 \mathrm{H}$, $\mathrm{CH}_{2} \mathrm{CH}_{3}$ ), 1.72 (q, $\left.J=7.2 \mathrm{~Hz}, 2 \mathrm{H}, \mathrm{CH}_{2} \mathrm{CH}_{3}\right), 2.51 \sim 2.45$ 
(m, 2H, $\left.\mathrm{SCH}_{2} \mathrm{CH}_{2} \mathrm{CH}_{3}\right), 3.13\left(\mathrm{t}, J=7.2 \mathrm{~Hz}, 2 \mathrm{H}, \mathrm{SCH}_{2}\right.$ ), $3.75\left(\mathrm{~s}, 3 \mathrm{H}, \mathrm{OCH}_{3}\right), 4.00\left(\mathrm{~d}, J=4.4 \mathrm{~Hz}, 2 \mathrm{H}, \mathrm{NHCH}_{2}\right)$, $6.74 \sim 6.64\left(\mathrm{~m}, 3 \mathrm{H}, \mathrm{C}_{6} \mathrm{H}_{3}\right), 6.80(\mathrm{~s}, 1 \mathrm{H}, \mathrm{NH}), 9.02(\mathrm{~s}, 1 \mathrm{H}$, $\mathrm{OH}) ;{ }^{13} \mathrm{C}$ NMR (101 MHz, DMSO- $\left.d_{6}\right) \delta: 157.1,150.5$, $147.8,146.7,127.6,122.3,115.7,113.9,56.0,54.7,40.5$ (d, $J=20.7 \mathrm{~Hz}), 40.4 \sim 40.3,40.1(\mathrm{~d}, J=17.7 \mathrm{~Hz}), 40.0$, 39.8, 39.6, 39.4, 34.0, 23.0, 17.7, 13.5, 11.5; MS (ESI) $m / z$ : $320.88[\mathrm{M}-\mathrm{H}]^{-}$. Anal. calcd for $\mathrm{C}_{15} \mathrm{H}_{22} \mathrm{~N}_{4} \mathrm{O}_{2} \mathrm{~S}: \mathrm{C} 55.88, \mathrm{H}$ 6.88, N 17.38; found C 55.84, H 6.83, N 17.41.

\section{3 化合物 $1 \mathrm{c}$ 的单晶测定}

将化合物 $1 \mathrm{c}$ 溶于无水乙醇, 微孔滤膜过滤, 滤液置 于安静的环境中, 室温下挥发结晶, 得到无色块状晶体. 挑选 $0.29 \mathrm{~mm} \times 0.24 \mathrm{~mm} \times 0.20 \mathrm{~mm}$ 大小的单晶, 使用 Bruker AXS SMART 1000 CCD X 单晶衍射仪, Mo k $\alpha$ $(\lambda=0.71073)$ 光源测定晶体结构, 在 273(2) K 下以 $\omega-\psi$ 扫描方式收集数据. 然后运用 Bruker 的 SAINTPLUS 程 序 ${ }^{[32]}$ 将数据还原, 同时运用 SADABS 程序 ${ }^{[33]}$ 进行经验 吸收校正. 应用 SHELXS-97 和 SHELXL-97 程序 ${ }^{[34]}$ 直接 法解析和精修结构. 所有的非氢原子采用全矩阵最小二 乘法进行结构精修. 所有非氢原子都做各向异性精修. 理论加氢, 氢原子各向同性热参数修正.

\section{4 目标化合物的 NA 抑制活性测定}

用 NA 的特异性荧光底物 2'-(4-甲基企形酮基)- $\alpha-D-$ $N$-乙酰神经氨酸(MUNANA; Sigma)来测定 NA 活性. 分 别用 365 和 $460 \mathrm{~nm}$ 的激发和发射波长测定 $\mathrm{NA}$ 与 MUNANA 作用后释放的 4-甲基-贝伐酮的苂光. 苂光 程度越高, 则 NA 活性越强, 根据荧光强度的变化计算 化合物对 NA 活性的抑制率.

将 $10 \mu \mathrm{L}$ 待测化合物溶液 $(40 \mu \mathrm{g} / \mathrm{mL}), 30 \mu \mathrm{L}$ 一定浓 度的神经氨酸酶液 $[\mathrm{A} / \mathrm{PR} / 8 / 34(\mathrm{H} 1 \mathrm{~N} 1)]$ 和 $10 \mu \mathrm{L} 33$ $\mathrm{mmol} / \mathrm{L} 2-(N$-吗啉代)乙磺酸 $(\mathrm{MES})$ 缓冲液 $(\mathrm{pH}$ 6.5)加入 96 孔板中, 混合均匀, $37{ }^{\circ} \mathrm{C}$ 条件下捊育 $15 \mathrm{~min}$. 加入 10 $\mu \mathrm{L} 4 \mathrm{mmol} / \mathrm{L}$ 的二氯化钲溶液、 $10 \mu \mathrm{L} 20 \mathrm{mmol} / \mathrm{L}$ 底物 MUNANA, 混合均匀, $37{ }^{\circ} \mathrm{C}$ 孵育 $30 \mathrm{~min}$. 加入 $150 \mu \mathrm{L}$ 终止液(氢氧化钠溶于质量分数为 $83 \%$ 的乙醇水溶液中, 配置成 $14 \mathrm{mmol} / \mathrm{L}$ 的终止液), 反应终止. 在激光波长 $360 \mathrm{~nm}$ 和发射波长 $450 \mathrm{~nm}$ 的条件下, 测定荧光强度. 根据荧光强度的减少量计算化合物对 NA 活性的抑制 率. 将抑制率大于 $50 \%$ 的化合物进行三次复笁, 根据复 笁结果计算化合物的 $\mathrm{IC}_{50}$ 值. 流感病毒 $\mathrm{A} / \mathrm{PR} / 8 / 34$ (H1N1)神经氨酸酶由中国疾病预防控制中心提供, 葛 兰素公司的扎那米韦(zanamivir)为阳性对照药.

\section{5 分子对接}

为深入研究活性化合物与 NA 的相互作用情况, 将
NA 抑制活性最佳的化合物 1b 与 NA (PDA entry: 3TI6) 的活性位点进行分子对接. 选用 LeDock (http://www. lephar.com/) 软件进行分子对接, 其结合位点设置为 $X_{\min }=-37.4, X_{\max }=-19.8, Y_{\min }=4.0, Y_{\max }=21.5, Z_{\min }=$ 12.0, $Z_{\text {max }}=32.2$. 对接结果用 PyMOL (http://pymol. sourceforge.net/)软件显示并作图.

致谢 中国医学科学院北京协和医学院药物研究所提 供生物活性测定.

辅助材料(Supporting Information) 化合物 $1 \mathrm{a} \sim 1 \mathrm{k}$ 和 $\mathbf{2 b} \sim \mathbf{2 c}$ 的 ${ }^{1} \mathrm{H} \mathrm{NMR} 、{ }^{13} \mathrm{C} \mathrm{NMR}$ 谱图和 $\mathbf{1 c}$ 晶体的结构数 据. 这些材料可以免费从本刊网站(http://sioc-journal. $\mathrm{cn} /$ )上下载.

\section{References}

[1] Williams, S.; Fitzner, J.; Merianos, A. Bull. W. H. O. 2014, 92, 60.

[2] Kobasa, D.; Jones, S. M.; Shinya, K. Nature 2016, 445, 319.

[3] Wang, P. C; Fang, J. M.; Tsai, K. C. J. Med. Chem. 2016, 59, 5297.

[4] Kakuta, M.; Kubo, S.; Tanaka, M. Antiviral Res. 2013, 100, 190.

[5] Leang, S. K.; Kwok, S.; Sullivan, S. G. Influenza Other Respir. Viruses 2014, 8, 135.

[6] Harrington, P. J.; Brown, J. D.; Foderaro, T. Org. Process Res. Dev. 2003, 8,86 .

[7] Zhao, X.; Jie, Y.; Rosenberg, M. Antiviral Res. 2012, 96, 91.

[8] LackenAy, A.; Aesselaar, T. G.; Daniels, R. S. Antiviral Res. 2018, 157, 38.

[9] Air, G. M. Influenza Other Respir. Viruses 2012, 6, 245.

[10] Tong, S.; Li, Y.; Rivailler, P.; Conrardy, C.; Alvarez Castillo, D. A.; Chen, L. M.; Recuenco, S.; Ellison, J. A.; Davis, C. T.; York, I. A.; Turmelle, A. S.; Moran, D.; Rogers, S.; Shi, M.; Tao, Y.; Weil, M. R.; Tang, K.; Rowe, L. A.; Sammons, S.; Xu, X.; Frace, M.; Lindblade, K. A.; Cox, N. J.; Anderson, L. J.; Rupprecht, C. E.; Donis, R. O. Proc. Natl. Acad. Sci. U. S. A. 2012, 109, 4269.

[11] Wang, K.; Fei, Y.; Wang, L. Eur. J. Med. Chem. 2017, 141, 648.

[12] Pires, C. D. M.; Drusano, G. L.; Adams, J. R. Eur. J. Pharm. Sci. 2018, $111,443$.

[13] Dai, X. Q.; Zhu, Y. B.; Wang, Z. Y.; Weng, J. Q. Chin. J. Org. Chem. 2017, 37, 1924 (in Chinese) (戴小强, 朱亚波, 汪洲洋, 翁建全, 有机化学, 2017, 37, 1924.)

[14] Shen, Z. H.; Sun, Z. H.; Becnel, J. J.; Estep, A.; Wedge, D. E.; Tan, C. X.; Weng, J. Q.; Han, L.; Liu, X. H. Lett. Drug Des. Discovery 2018, 15, 951.

[15] Chen, L.; Shen, Z. H.; Xu, T. M.; Tan, C. X.; Weng, J. Q.; Han, L.; Peng. W. L.; Liu, X. H. J. Heterocycl. Chem. 2018, 55, 946.

[16] Zhai, Z. W.; Wang, Q.; Shen, Z. H.; Tan, C. X.; Weng, J. Q.; Liu, X. H. Chin. J. Org. Chem. 2017, 37, 232 (in Chinese). (翟志文, 汪乔, 沈钟华, 谭成侠, 翁建全, 刘幸海, 有机化学, 2017, 37, 232.)

[17] Liu, X. H.; Zhao, W.; Shen, Z. H.; Xing, J. H.; Xu, T. M.; Peng, W. L. Eur. J. Med. Chem. 2017, 125, 881.

[18] Li, Q. M.; Pang, K. S.; Zhao, J. P.; Liu, X. H.; Weng, J. Q. Chin. J. Org. Chem. 2017, 37, 1009 (in Chinese). (李倩梅, 庞凯胜, 赵建平, 刘幸海, 翁建全, 有机化学, 2017, 37,1009 .)

[19] Shi, J. J.; Ren, G. H.; Dai, Z. M.; Wu, N, J.; Weng, J. Q.; Xu, T. M.; Liu, X. H.; Tan, C. X. Lett. Drug Des. Discovery 2018, 15, 15.

[20] Wang, Y. R.; Zheng, D. D.; Wang, Y.; Ye, H.; Yao, W.; Ding, Y. Chin. J. Org. Chem. 2019, 39, 2053 (in Chinese). (王誉蓉, 郑丹丹, 王杨, 叶浩, 姚炜, 丁颖, 有机化学, 2019, 39, 
2053.)

[21] Ezabadia, I. R.; Camoutsisa, C.; Zoumpoulakisb, P.; Geronikakic, A.; Sokovićd, M.; Glamočilijad, J.; Ćirićd, A. Bioorg. Med. Chem. 2008, 16, 1150 .

[22] Pardeshi, S.; Bobade, V. D. Bioorg. Med. Chem. Lett. 2011, 21, 6559.

[23] Upmanyu, N.; Gupta, J. K.; Shah, K. J. Pharm. BioAllied Sci. 2011.

[24] Liu, X. H.; Pan, L.; Weng, J. Q. Mol. Diversity 2012, 16, 251

[25] Abou-Seri, S. M. Eur. J. Med. Chem. 2010, 45, 4113

[26] Sharma, V.; Mehta, D. K.; Das, R.; Kaur, P. K. Int. J. Pharm. Pharm. Sci. 2016, $8,28$.

[27] Liu, X.; Clercq, E. D.; Aalzarini, J. Lett. Drug Des. Discovery 2013, 10, 27.

[28] Kamel, M. M.; Megally, N. Y. Eur. J. Med. Chem. 2014, 86, 75
[29] El-Husseiny, W. M.; El-Sayed, M. A.-A.; Abdel-Aziz, N. I.; ElAzab, A. S.; Asirig, Y. A. Eur. J. Med. Chem. 2018, 158, 134.

[30] Liu, L.; Ye, J.; Xiao, M. W.; Yuan, K. Y; He, M.; Hu, A. X. J. Heterocycl. Chem. 2019, 56, 2192.

[31] Yang, Q. C.; Sun, X. H.; Liu, Y. F. Chin. J. Struct. Chem. 2015, 34, 197.

[32] Sheldrick, G. M. SADABS, Program for Empirical Absorption Correction of Area Detector Data, University of Gotingen, Germany, 1996.

[33] Sheldrick, G. M. SHELXTL V5.1, Software Reference Manual, Brucker AXS, Inc., Madision, Wisconsin, USA, 1997.

[34] Wang, X. F. M.S. Thesis, Nankai University, Tianjin, 2014 (in Chinese).

(王晓芳，硕士论文，南开大学，天津，2014.)

(Cheng, F.) 